\title{
THE CROSS-NATIONAL PATTERN OF HAPPINESS: Test of predictions implied in three theories of happiness
}

\author{
Ruut Veenhoven with the assistance of Joop Ehrhardt
}

Published in: Social Indicators Research, 1995, vol 34, pp 33-68.

\begin{abstract}
Predictions about level and dispersion of happiness in nations are derived from three theories of happiness: comparison-theory, folklore-theory and livability-theory. The predictions are tested on two cross national data-sets: a comparative survey among university students in 38 nations in 1985 and a collection of comparable general population surveys in 28 nations around 1980. Most predictions of comparison-theory and folklore-theory are defied by the data. The predictions of livability-theory are all confirmed.
\end{abstract}

\section{INTRODUCTION}

Happiness is a highly valued goal in social policy. It is generally agreed that we should try to reduce human suffering and make life more satisfying for a greater number. As such, happiness is a current output-indicator of policy success (Veenhoven, 1993A).

Though there is wide agreement that we should promote happiness, there is doubt whether we can do much about it. This doubt is embodied in two theories of happiness: 'comparisontheory' and 'folklore-theory'. Both these theories predict that a better society makes no happier people. They contradict common sense 'livability-theory', which holds that improvement of living-conditions in a society will make life more enjoyable.

Below I will consider these theories in more detail and confront them with data of two cross-national surveys on happiness.

\section{THREE THEORIES OF HAPPINESS}

Happiness or life-satisfaction is the degree to which one judges the quality of ones life favourably. Elsewhere I have delineated this definition in more detail Veenhoven, 1984: 2225 ). Theories of happiness differ in assumptions about the sources of information used in the evaluation of life.

\subsection{Comparison-theory}

This theory assumes that the evaluation of life is based on a mental calculus, in which perceptions of life-as-it-is are weighted against standards of how-life-should-be. Standards of comparison are presumed to be variable rather than fixed, and to follow perceptions of possibilities. In other words, we would judge life by what we think it can realistically be.

What life could possibly be is either inferred from observation of other people, or by our own experiences. Therefore, improvement of living conditions in a country would inevitably involve a raising of standards. Conversely, deterioration of living conditions would lower 
standards of comparison. Because standards follow success, the difference between standard and reality remains the same in the long run. As a result, subjective appreciation of life would be unrelated to the objective quality of it.

There are two main variants of this theory: 'social -comparison' and 'lifetime-comparison'.

The social-comparison variant stresses comparison with other people. It holds that people will be unhappy in spite of good conditions if they compare with others who are in an even better situation. Likewise, people would be happy in adverse conditions if they compare with others who suffer even more.

The lifetime-comparison variant presumes that we judge our life in the cognitive context of our best and worst experiences. This variant claims that people will be unhappy in good conditions if they happen to have enjoyed even better before. Conversely, people would be happy in adverse conditions if life was even worse before.

In both variants relative deprivation determines happiness; not absolute deprivation.

Comparison theory of happiness has been advocated by Brickman and Campbell (1971) and by Easterlin (1974). Elsewhere I have discussed the assumptions of this theory in more detail (Veenhoven, 1991).

\subsection{Folklore-theory}

This theory does not see happiness as an individual evaluation of life, but as the reflection of a body of widely held notions about life, that is part of the national character. These notions are seen to root in tradition, rather than in current realities of life. Consequently, subjective appreciation of life in a country is expected to be largely unrelated to the present quality of life in that country.

An example may illustrate this theory: If hardship in earlier generations has brought on a pessimistic outlook on life in a country, that outlook may persist for quite some time, and will discourage a positive judgment of life in later generations. As a result a later generation can be largely dissatisfied with life, even if living conditions have in fact become quite favorable. Inglehart (1990:30) suggests this is the case in France and Italy. Conversely, a culture of optimism is seen to produce a fairly high level of happiness in spite of rather miserable living conditions. The case of the USA is often mentioned in this context (e.g. by Ostroot and Snyder 1985).

Folklore-theory is the collective variant of 'trait-theory' of happiness. Elsewhere, I have considered this theory in more detail (Veenhoven, 1993B, 1994).

\subsection{Livability-theory}

This is the theory that subjective appreciation of life depends in the first place on the objective quality of life; the better the living-conditions in a country, the happier its inhabitants will be. Unlike comparison-theory, livability-theory focuses on absolute quality of life, rather than on relative difference. People are presumed to be happy in good living-conditions even if they know that others enjoy even better conditions.

'Good' living-conditions are presumed to be conditions that fit human nature well; in other words, living-conditions that are 'livable'. For most animals livability is largely an ecological matter, for the human species societal qualities are involved as well. The livability of one's society is the degree to which collective provisions and demands fit with individual needs and capacities. Factual requirements for livability do not necessarily coincide with desirability's of 
ideology. Elsewhere I have delineated this concept in more detail (Veenhoven, 1993B: ch 2).

Livability-theory is closely connected to the idea that there are universal human needs. It sees human societies as collective arrangements to gratify these needs, and assumes that societies can be more or less effective in that respect. The theory is a basic canon in social policy and hence in Social Indicators Research. It was in fact taken for granted until various studies in western nations showed that subjective happiness is hardly related to objective position with respect to income, education, age and gender.

\section{IMPLIED PREDICTIONS ABOUT NATION DIFFERENCES}

These three theories imply some predictions about cross-national differences in happiness, both about its level and its dispersion. They also involve some predictions about differences in happiness between social categories within countries. The predictions are contradictory in several cases.

\subsection{Predictions About Level of Happiness in Nations}

The three theories have different implications for the characteristic degree of happiness to be expected in most of the countries in this world; whether people will be typically happy, neutral or unhappy. The theories also differ in the cross national variation they suggest; whether or not people will be equally happy or unhappy in all countries of this world. If people appear not to be equally happy everywhere, the differences in average happiness can either be related to variation in quality of life in the country or not. The theories differ in predictions about that latter matter as well.

\subsubsection{Comparison-theory}

Prevalent level: Comparison-theory predicts that the prevalent level of happiness in most countries of the world will be around neutral. Different variants of the theory predict so for different reasons.

The social-comparison variant presumes that we will be happy if we think we are better off than others, and unhappy if we think we are worse off. In that line of thought, average happiness in a country depends on reference behavior; the more downwards the comparisons, the more happy the average citizen in a nation. If we do not make further assumptions about that matter, we assume in fact that reference is random. That most basic form of the theory implies that average happiness will be typically neutral. If reference is random, the chance of comparing to people who are better off is equally great as comparing to people who are worse off. Most people will then be neutral about their life, because positive comparisons outweigh negative ones. In the remaining part of the population the happiness of the ones who compare positively will balance the unhappiness of the ones who happened to compare negatively. Hence the nation average will be neutral as well.

A common additional assumption also implies that average happiness will be neutral. That is the above mentioned assumption is that reference follows success and failure. People who feel better off, will then not feel so forever, because they stop comparing with the less well off. In that view, happiness is a short lived state that tends to fluctuate around the neutral state of perceiving oneself as neither better off nor worse. The average happiness of a person during his or her life will therefore be about zero. Consequently, the average level in the nation will be also zero. 
In fact, social comparison can explain a non-neutral average only by introducing accessory premises which are farther away from its core; for instance the assumption that we tend to downward comparison (which implies that a positive appreciation of life must be the rule), or the assumption that we compare upward with the salient jetset (which predicts that the mass is characteristically unhappy).

The lifetime-comparison variant of the theory holds that we judge our life on the basis of earlier experience. We would be happy if we think we live better than before and unhappy if we perceive a move back. Again, it is the difference that counts; not the actual level of living. If we do not make further assumptions, the chance of being better off or worse than before is again a random matter. The result is once more that most people will be neutral about their life and that the happy and unhappy balance out. As such, this variant also suggests that average appreciation of life will be typically neutral in nations. The basic premise of the theory does not embody a reason to expect that a positive or a negative appreciation of life will be the rule.

Also in this case, common accessory assumptions suggest the same outcome. One such assumption is that most people remain essentially at the same level of living during their lifetime, which implies that most people will consider themselves neither happy nor unhappy. Another current assumption is that we orient on the most salient top- and bottom experiences in life, and for that reason tend to experience everyday life as mediocre. These effects will also produce an average about neutral in nations.

However, as in the case of social-comparison, we can also think of adjunct assumptions that predict a non-neutral outcome; e.g., the assumption that we remember adverse life-events better than good ones (and for that reason tend to be happy), or conversely, that we typically enjoyed a happy youth (and for that reason tend to be rather unhappy in adulthood).

Let it suffice to note that the core premises of comparison-theory suggest that average happiness tends to be neutral. This implication is known as 'zero-sum' theory (Unger 1970).

Similarity across nations: By implication, comparison-theory also predicts that differences in average happiness between nations will be small. If average happiness tends to be zero, it must be around zero everywhere. Differences in nation averages must then be in the limits of chance variation.

Most of the above mentioned additional assumptions also imply that the level of happiness will be about the same in all countries of the world, even the premises that predict a non-zero outcome. All assumptions about cognitive tendencies in comparison, such as downward comparison or salience of past adversity, are likely to manifest universally and hence produce a similar outcome in all countries.

That prediction is also implied in the common adjunct assumption that we compare in the first place with 'similar' people. That means that compatriots will compare with compatriots, and that countries serve as separate reference-units. Easterlin (1974) concludes on that basis that happiness must be about the same in all countries of the world

The explanation of possible differences in happiness between nations would require accessory premises, which are fairly remote from the core theory.

Correspondence with QOL in nations: If nevertheless there are sizable differences in average happiness across nations, comparison theory suggests that these differences bear little relationship with the quality-of-life. As we have seen above, this theory implies that subjective happiness can be high in adverse conditions and low in good ones.

The social-comparison predicts so on the basis of the above mentioned assumption that we compare to compatriots rather than to aliens. On that basis Easterlin (1974) claimed that 
happiness is essentially unrelated to quality-of-life in the country. However, if we assume that people compare across borders and that their perceptions of differences are realistic, socialcomparison-theory does predict some correspondence.

The lifetime-comparison variant is less ambiguous in this matter. If our personal best and worst experiences frame the judgment in the first place, there will be little or no relationship with the nations quality-of-life.

Taken together, comparison-theories predict little or no correspondence between level of happiness and quality-of life in nations.

\subsubsection{Folklore-theory}

Prevalent level: Folklore theory does not imply a prediction about the typical degree of happiness reported in the present day world. According to this theory, average happiness in a country depends in the prevailing outlook on life. In itself, the theory does not involve reasons to assume that views on life are typically positive or negative in the present day world. Neither are there common adjunct assumptions to this theory that suggest so.

In this case there is less chance that random variation presses to the neutral level. We deal with a limited number of cultures, rather than with the enormous amounts of individuals in the earlier case of comparison-theory.

Similarity across nations: If there is variation in characteristic outlook on life between nations, there must also be variation in average happiness. In its most basic premise, the theory does not involve a prediction on whether the difference will be big or small. Folklore could be rather similar everywhere, or different.

However, proponents of the theory typically see the national character as the result of unique cultural and historical constellations and assume this results in profound differences in outlook on life. These secondary premises imply that average happiness will be different across nations.

Folklore-theory can predict similarity in happiness only when rather remote assumptions are introduced; for instance, that globalization has erased differences in outlook on life.

Correspondence with QOL: Folklore-theory further suggests that differences in average happiness in nations will be largely unrelated to variation in actual quality of life. It is not the reality of life that matters, but the cultural glasses through which one looks at it. A positive outlook could make people happy in spite of miserable living conditions, while gloomy outlook might create unhappiness in Paradise.

\subsubsection{Livability-theory}

Prevalent level: Livability-theory does not allude to a certain level of happiness in its basic premise. Average happiness in a nation depends on the livability of society, and that livability can be either good or bad. Again the number of nations is too small to expect that random variation in livability will result in a tendency to the neutral.

A reasonable additional assumption would seem that present day societies are fairly well livable; the human species having evolved in comparably austere living conditions. Happiness can then be expected to be typically above neutral in the present day world. However, one can also assume that modem 'Gesellschaft' fails to provide the socio-emotional support of traditional 'Gemeinschaft' and for that reason predict that happiness will be below neutral. 
The former assumption being equally plausible as the latter, we must conclude that livabilitytheory does not predict that either happiness or unhappiness is the rule.

Similarity across nations: Because livability can be either good or bad, the theory suggests that the level of happiness is characteristically different across countries. Similarity in happiness can fit this theory only if the further assumption is made that present day nations are about equally livable; e.g., as the result of convergence in an emerging world society.

Correspondence with $Q O L$ : Obviously, the theory predicts a strong relationship between average happiness of the citizens and the degree of livability of the country. If observed correlations are less than perfect, this must be due to measurement problems.

The various predictions about level of happiness in nations are summarized in Scheme I; upper half.

\subsection{Predictions About Dispersion of Happiness Within Nations}

Next to the level of happiness in a country, we can also consider its dispersion. The highest possible degree of dispersion is when half the population is very happy and the other half very unhappy; the lowest possible level is when all citizens are about equally happy. Dispersion of happiness within nations can be characteristically high, medium or low. Across nations, dispersion can be either similar or dissimilar. If dissimilarity is the rule, differences in dispersion can either be related to living-conditions in the country or not; in particular to the degree of inequality in life-chances between citizens. The three theories differ also in their predictions on these matters.

\subsubsection{Comparison theory}

Prevalent dispersion: Comparison theory implies that the degree of dispersion will be characteristically low. This follows from the above discussed inferences that happiness tends to fluctuate around neutral, and that we feel neutral most of the time. In this view the observed dispersion must be close to random fluctuation.

Similarity across nations: For the same reason, comparison theory predicts that dispersion of happiness within nations is highly similar across nations. If dispersion is typically low, it must be low everywhere.

Correspondence with inequality: At first sight, comparison-theory would seem to suggest that dispersion of happiness will be greater in nations where differences in life-chances are greatest; differences in life-chances creating differences in happiness. However, on second look we must realize that comparison theory is not about absolute differences, but on relative differences. It is the difference to comparison-standard that matters, rather than the factual difference in life-chances.

In the social-comparison variant of the theory, social inequality in a country does not necessarily result in comparison with dissimilar people. In a class-society, people can compare in the first place with members of their own class. People of all ranks are then about equally happy and the dispersion of happiness thus low. Even if people compare across classes, dispersion can still be low. Suppose that all citizens in a country tend to compare with 
an advantaged elite who sets the standards by conspicuous consumption and by domination of the media. The great majority will be unhappy in that country and the elite neutral. Dispersion of happiness will be low in that case, given the concentration at the lower end of the scale. In fact, social-comparison theory requires additional assumptions explain higher dispersion of happiness in socially unequal countries; e.g., a split in society involving equally sizable classes of advantaged and disadvantaged (to avoid the inference of mass unhappiness), and downward comparison of the advantaged class (to explain that the latter are very happy rather than neutral).

The lifetime-comparison variant does not predict that dispersion of happiness will be greater in unequal society. Though social inequality may create greater dispersion of good and bad life-experiences in society, it does not necessarily bring greater differences between bestand worst experiences at the individual level. Even if life for the disadvantaged is worse in an unequal society, the difference between best and worst experience may still be the same.

All in all, comparison-theory predicts little or no correspondence between dispersion of happiness and social inequality in nations.

\subsubsection{Folklore-theory}

Prevalent dispersion: Folklore-theory predicts that the degree of dispersion will be typically low. Because all citizens in a country look at their life though the same glasses, their level of happiness is likely to be similar. High dispersion fits this theory only if additional premises are introduced; for instance, assumptions about sub-cultural differences in outlook.

Similarity across nations: By implication, folklore-theory also predicts that dispersion of happiness in nations will be highly similar between nations. If the dispersion of happiness in countries is typically low, nations are unlikely to differ very much in this respect.

Correspondence with inequality: If there are still differences in dispersion of happiness, folklore-theory suggests that these differences bear little or no relationship with social inequality in the country. Because a preconceived outlook dominates the evaluation of life, differences in life-chances between compatriots will affect happiness only to a limited extent.

\subsubsection{Livability theory}

Prevalent dispersion: Livability-theory holds that the dispersion of happiness in a country can be either high or low, depending on differences in living-conditions between compatriots. Hence the theory does not involve a prediction about a universal degree of dispersion.

Similarity across nations: The theory does suggest that dispersion of happiness will be dissimilar across nations. Similarity in degree of dispersion can fit the theory only if the secondary assumption is made that life-chances tend to be equally unequal in all nations of the present day world. That assumption is highly improbable.

Correspondence with inequality: Livability-theory also predicts that differences in dispersion across nations are closely related to variation in social inequality. The more unequal lifechances are in a country, the greater the differences in happiness of its citizens.

The above predictions about dispersion of happiness in nations are summarized in the lower half of Scheme I. 


\subsection{Predictions About Differences in Happiness of Students and the General Population}

The cross-national pattern of happiness may be different if we consider specific social categories within nations. For instance, nation-differences in level of happiness could be less pronounced when aged persons are compared rather than average citizens. The three theories suggest different reasons why this could be so; more uniform reference in comparison-theory, greater similarity of outlook in folklore-theory and more equality of living conditions in livability-theory.

There is a good data-set on cross-national differences in happiness among university students. Part of that set allows comparison with a collection of general population surveys on happiness. These data will be discussed in the next paragraph. Here I will consider the possible differences the three theories suggest. I derive predictions about divergent patterns among students on the basis of the assumption that students typically differ from the general population, in that their cultural orientation and living conditions are more homogenous within nations and more similar across nations.

\subsubsection{Differences in level of happiness between students and the general population}

Differences in average happiness: Comparison-theory suggests that students will be about equally happy as the general population. The above mentioned implication, that happiness tends to the neutral, applies to the former as well as to the latter. Moreover, the common assumption of social comparison to 'similar' people implies that happiness is about the same in each reference group (the same reason why happiness would be similar across nations).

Folklore-theory rather suggests that there are slight differences in level of happiness between students and the general population. On the one hand students live in the same culture and therefore look at life in a similar way. On the other hand students have more contact with current academic world culture, and may therefore differ in outlook from local views in some respects. The difference may result in more or less happiness. The theory does not predict the direction of the difference.

Livability-theory suggests slight differences as well. In this theory, average happiness is different if the quality of life is different. Because students and the average citizen live in the same country, living conditions are at least partly similar. Still, there may be differences; in some countries student life can be relatively harsh for students and in other countries comparatively lax. Hence, students can be somewhat less or more happy.

Similarity across nations in the pattern of difference: Comparison-theory suggests that the pattern of difference will be the same in all countries of the world. Because it predicted that there is typically no difference in average happiness between students and the general population, it implies that the non-difference will be the same everywhere.

Folklore-theory did suggest that average happiness can differ slightly between students and the general population. It also suggests that the pattern of difference varies across nations, nation differences in happiness of students being smaller than nation-differences in happiness of the general population. If university students are more oriented on a world wide academic culture, they are more likely to look at their life in the same way, and be equally happy as a result.

Livability-theory also suggests less differences among students. The above assumption of 
greater similarity in living conditions implies greater similarity in average happiness.

Correspondence with QOL in the pattern of difference: As we have seen, comparison-theory suggests that students tend to be equally happy (neutral) as the average citizen in their country, and that this pattern of non-difference is universal. If nevertheless there are differences, and variation in that across nations, the theory would suggest that these nationdifferences in student/citizen-differences have little to do with the quality of life in the nation. If such differences exist at all, these must be due to cultural variation in categorial reference behavior in the first place.

Folklore-theory does not rule out a difference, but also predicts that the pattern of difference in nations will be unrelated to the quality of life in these nations. If there is a difference in happiness between students and the general population, that is due to outlook on life, rather than to quality of life.

Livability-theory does suggest that the pattern of difference corresponds with quality of life in nations. As we have seen earlier, this theory predicts that average happiness in nations is closely related to quality of life. With respect to the difference between students and average citizens, it further suggests that correlations will be less pronounced among students. This follows from the assumption that cross-national differences in living-conditions are smaller among students.

\subsubsection{Differences in dispersion of happiness between students and the general population}

Difference in degree of dispersion: In itself comparison theory does not involve reasons to suggest that dispersion of happiness will be smaller or greater among students than among the general population. However, some fairly plausible additional assumptions predict greater dispersion among students.

In the social-comparison variant, dispersion of happiness in a group living in similar conditions must be the result of variation in reference. University students have relatively more choice of reference groups; they can compare either with their less successful age mates, with fellow students and with established professionals. Reference behavior is also likely to change more among students than among the average citizen. As such, we can expect somewhat more dispersion of happiness among students.

In the life-time variant, dispersion of happiness is greater, the more persons in a population feel currently better or worse than ever before. Such extreme experiences will occur more often among the young than among the old. The more life-experiences, the greater the chance that the next will be within the limits of earlier experience. Consequently, dispersion of happiness must be greater among students than in the general population.

Folklore theory rather suggests that dispersion of happiness tends to be lower among students. This follows at least from the assumed greater cultural homogeneity in this category. If students are more similar in outlook on life than their compatriots, they must diverge less in their evaluation of it.

Livability-theory also suggests that dispersion will be lower among students. Here that inference follows from the assumption that living conditions of students tend to be more homogenous.

Similarity across nations in the pattern of difference. Comparison-theory suggests that the difference between students and the general population will be similar in all countries of the world. The above mentioned reasons to expect somewhat more dispersion among students apply everywhere.

Folklore-theory rather suggests that the pattern of difference will vary across nations. Differences in outlook on life between students and the average citizen are not the same 
everywhere. If we assume that views diverge more in the developing countries than in the developed ones, folklore-theory predicts greater difference in dispersion in the former countries than in the latter.

Livability-theory also suggests that the pattern of differences will be variable across nations. Because relative homogeneity of living conditions is not the same everywhere, the difference in dispersion of happiness is neither.

Correspondence with social inequality in the pattern of difference: If dispersion of happiness among students differs from dispersion in general population samples, and if the difference is not identical in all nations, there can either be a pattern in the nation-differences or not. As we have seen above, differences in dispersion of happiness are likely to correspond with nationvariation in social inequality.

Comparison-theory does not suggest that a possible difference in dispersion of happiness between students and average citizens will depend on the degree of inequality in the country. As we have seen this theory predicts that there is no difference at all.

Folklore-theory does suggest that dispersion of happiness differs between students and general population samples, and that the pattern of difference can vary across nations. However, it does not imply that the difference will be greater in nations that stand out by unequal life-chances. If there is any systematic nation-difference at all, the theory would rather suggest that the difference is greater in cultural heterogeneous countries, where the dispersion of happiness among the general population will be relatively great.

Livability-theory also suggests that there is a difference, and that the pattern of difference varies with social inequality in the nation. As we have seen above, this theory predicts that dispersion of happiness will be greater in the most unequal nations. In this context, it further predicts that dispersion of student-happiness corresponds less with social inequality in the country than dispersion of happiness in the general population. This follows from the assumption of greater cross-national homogeneity in living-conditions among students.

The various predictions about differences in the pattern of happiness between students and average citizens in nations are summarized in Scheme II.

\section{DATA}

Two cross-national data-sets are considered: a comparative survey among university students in 39 nations around 1985 and a collection of comparable surveys among general population samples in 28 nations around 1980.

The survey among university students was part of an attempt to test Michalos' (1985) Multiple Discrepancies Theory cross-culturally. The questionnaire involved two single items on subjective appreciation of life. One question was "How do you feel about your life as a whole right now?" and was answered on a 7-step rating-scale ranging from terrible (1) to delightful (7). This is referred to as the 'life-satisfaction' item. The other question was "Considering your life as a whole, would you describe it as very unhappy (1), unhappy (2 or 3 ), mixed (4), happy (5 or 6) or very happy (7). This item is referred to as 'happiness'.

Data were obtained from 18032 students in 39 nations. Questionnaires were administered in classroom, mostly in social science courses. The data are therefore not quite representative of the national student populations. This data-set is fully described in Michalos (1991).

Average scores per country on the two questions are presented in Appendix A, the standard-deviations in Appendix B.

The collection of representative nation samples was drawn from Veenhoven's (1993) World Database of Happiness. This database contains the results of survey studies that involved 
acceptable questions on happiness.

Studies were selected that concern the same period and used comparable items. Around 198028 studies are available that involved comparable questions about 'happiness-in-life'. Because the items differ slightly in wording and scale, the responses transformed scores. See Veenhoven 1993: ch 4 for the details. In similar way responses to questions on 'satisfactionwith-life' in the same period was harmonized. In order to facilitate comparison, the general population data were downsized linearly to scale $1-7$.

The general population averages are also presented in Appendix $\mathbf{A}$ and the standarddeviations in Appendix B; both in the right columns.

\section{RESULTS}

\subsection{Level of Happiness in Nations}

On the 7-step scales in the student-survey, average happiness in a country can maximally be 7 and minimally 1 . This is of course unlikely to occur. Given inevitable human suffering and random effects in reports of happiness, we can hardly expect that a nation-average will ever be higher than 6. Likewise, it is hard to image that average happiness can be lower than 2 in a country. The value of 4 is neatly in between. This is the neutral point in both rating-scales.

The scales used in the general population surveys are not symmetrical and lack a neutral answer-category. According to expert-ratings, the neutral point on these scales is also about 4, when transformed linearly to scale $1-7$. (See Veenhoven 1993: 127/130).

Prevalent level: In Appendix A we can see that the average scores on happiness and lifesatisfaction clearly above neutral. Among students the averages are respectively 4.63 and 4.81. Inspection of the general population samples yields a similar result: average happiness is 4.77 and average satisfaction 5.14. All these averages are significantly different from neutral $(\mathrm{p}<0.01)$. Mind that the data-sets do not quite coyer the same countries, and that neither is representative of the present day world.

These results are contrary to the zero-sum prediction implied in corn pan son -theory.

Differences across nations Appendix A also allow an examination of similarity in average happiness across nations. In the student survey, scores on the happiness item vary between 4.02 (Cameroon) and 5.27 (USA). The actual range is thus $21 \%$ of the maximally possible range. Average scores on the life-satisfaction item vary between 4.09 (Japan) and 5.44 (Finland). Here the actual range is $22 \%$ of the theoretical range.

Among the general population samples the differences are even greater. Average happiness varies between 4.09 (India) and 5.68 (Sweden). This is 28\% of the possible range. Satisfaction varies between 3.01 (again India) and 6.16 (Switzerland). This is 53\% of the possible range.

If the differences are expressed in the realistically possible range $2-6$, rather than the theoretical range $1-7$ the variation is between $30 \%$ and $80 \%$. All in all we can reject the hypothesis that happiness is essentially similar in all countries of the world. This is in line with livability-theory and folklore-theory, but contrary to comparison-theory.

Correspondence with QOL: The data of the student survey are presented on the left half of Scheme III. Average happiness is crossed with quality of some major living-conditions in the country. Sizable positive correlations appear. Students are clearly happier in the countries that are economically most affluent, politically most free and that provide most access to knowledge. The relationship with social equality is less clear, equal rights for women goes 
with greater happiness, but income-equality does not.

Of only 18 nations in the student-set do we have complete information on all quality of life variables. In that subset these variables explain together $53 \%$ of the cross-national variance in average happiness and $45 \%$ of the variance in average life-satisfaction.

The same analysis was done on the general population samples. The results are largely identical. Together the quality of life variables explain $78 \%$ of the nation-differences in average happiness and $63 \%$ of the variance in average life-satisfaction. These data are discussed in more detail in Veenhoven 1993: 50.

Clearly, there is a close relationship between objective quality-of-life and subjective happiness. This is contrary to the above derived predictions of comparison theory and folklore-theory, but in line with the core prediction of livability-theory.

\subsection{Dispersion of Happiness in Nations}

On the 7-step rating scales used in the student study, the maximally possible standarddeviation is 3 (50\% of the scores 1 and $50 \%$ score 7$)$. This is of course too extreme to exist in reality. The minimally possible $\mathrm{SD}$ is 0 . This requires that all responses in a country are in the same category; either positive, neutral or negative. This is neither likely to occur in reality. The realistically possible variation in dispersion of happiness on this $1-7$ scale will between about SD 0.5 to SD 2.5.

It is difficult to say what degree of dispersion is to be called 'small' or 'great'. Unlike the above case of averages, we have no clear neutral point here. By lack of a better criterion I will again take the midpoint of the possible range as a reference. I will consider standarddeviations around 1,5 as 'normal', below 1 'small' and above 2 'great'.

Dispersion in the student survey can not just be compared with the dispersion in the general population surveys. The latter used scales of a different length. The length of a ratingscale can possibly influence the dispersion of responses. In the general population surveys happiness was mostly measured by 4-step items. Standard-deviations were linearly transformed to scale $1-7$. Life-satisfaction was mostly rated on $1-10$ or $0-10$ scales. Standard-deviations were downsized linearly to scale $1-7$. The data are presented in Appendix B. Standard-deviations of happiness and life-satisfaction tend to be very similar in all nations; both among students and in general population samples. That latter result suggests that the variation in length of rating-scales did not involve major distortion.

Prevalent dispersion: In the student-survey the average standard-deviations in nations are 1.14 for happiness and 1.01 for life-satisfaction. In the general population samples the mean standard-deviations are respectively 1.37 and 1.28. These values cannot be characterized as 'small'; in particular not the latter. This result is contrary to the predictions of comparisontheory and folklore-theory.

Similarity across nations: In the student-survey, the smallest dispersion in responses on the happiness-item is observed in Greece (SD 0.92) and the greatest in India (SD 1.43). The difference is $17 \%$ of the theoretical range. Dispersion in responses on the life-satisfactionitem is lowest in Belgium (SD 0.80) and greatest in South Korea (SD 1.50). The difference is here $23 \%$ of the possible range.

In the general population samples nation differences in dispersion are even greater. Dispersion in responses on the happiness-item is smallest in Singapore (SD 0.99) and greatest in Greece (SD 2.01). The difference is $34 \%$ of the possible range. On the life-satisfaction-item 
the smallest dispersion appears in Australia (SD 0.88) and the greatest in Italy (SD 1.68). This latter difference is $27 \%$ of the possible range.

When expressed in the realistically possible range of $0.5-2.5$ rather than the theorical range $0-3$, the variation is $25 \%$ to $50 \%$. Though the difference is dispersions of happiness are somewhat smaller than the differences in average level, we can again reject the hypothesis of similarity across nations. That result does not fit the predictions of comparison-theory and folklore-theory, but accords with the prediction of livability-theory.

Correspondence with inequality: In Scheme IV the differences in dispersion of happiness in nations are crossed with some indicators of social inequality in nations.

In the student sample, dispersion of happiness appears to be unrelated to social inequality. Though all in the predicted direction, none of the correlations is significant. The explained variance is only $18 \%$. Analysis with SD-life-satisfaction yields a similar result (data not shown).

In the general population the correlations are more sizable. All indicators, except incomeinequality, are significantly related with dispersion of happiness. Together the four indicators of social inequality used here explain $63 \%$ of the variance. Analysis with the life-satisfaction data on a smaller number of nations yielded far smaller correlations, and an explanation of only $38 \%$ of the variance (data not shown).

The correlations observed in the general population samples contradict the predictions derived from comparison-theory and folklore-theory. Again the data fit better with livabilitytheory. When the non-correlation among student scores is interpreted in the context of the latter theory, it would seen that social inequalities in nations are reflected less among students than among the general population. That is in line with the above advanced assumption of greater homogeneity in the living conditions of students.

\subsection{Difference in Pattern Among Students and Average Citizens}

Data on the happiness of both students and the general population are available for 23 countries. These data are presented graphically in Scheme V (level) and Scheme VI (dispersion).

Difference in level of happiness: Scheme $\mathbf{V}$ shows that students tend to be somewhat less happy than the general population in the 23 nations considered here. On the happiness-item students score 4.85 and the general populations 5.19 . The differences are $6 \%$ of the possible range. Mean responses on the life-satisfaction-item are respectively 4.87 and 5.08, which is about $4 \%$ of the possible range. The differences are statistically not significant, so we cannot reject the hypothesis that there is 'no' difference as implied in comparison-theory. However, neither can we reject the hypothesis that there are 'slight' differences, as predicted by folklore-theory and livability-theory. Hence this test ends undecided.

Though the differences in overall-means are small, there are sizable differences at the country level. For instance, in Japan students are clearly less happy than the general population, whereas in India students are much more happy. This pattern is reflected in rather modest correlations between happiness of students and general populations; $r=+0.49$, respectively +0.56 . These results do not fit the prediction of comparison-theory of a similar pattern of non-difference in all nations. The data rather support the predictions implied in folklore-theory and livability-theory. In line with these theories, student-happiness is more similar across nations than the happiness of average citizens. This appears in the dispersions of the means: SD's of student-averages and general-population-averages are respectively 0.31 
and 0.41 in the case of happiness, and 0.36 and 0.61 in the case of life-satisfaction.

Scheme III has already shown that average subjective happiness in nations corresponds with objective quality of life. The correspondence is slightly lower in the student-survey than in the general population data-set. This result is in line with livability-theory, but contradicts the similar pattern of non-correlation predicted by comparison-theory and livability-theory.

Differences in dispersion of happiness. Scheme VI shows that the dispersion of happiness is somewhat lower in the student samples than in the general population samples. Mean standard-deviations on the happiness-item are respectively 1.14 and 1.37 , which is $12 \%$ of the possible range. On the life-satisfaction-item mean SD's are 1.01 and 1.28 , which is a difference of $10 \%$ of possible range. The differences do not meet the 0.05 level of statistical significance $(\mathrm{p}<0.10)$. This result is contrary to the prediction of greater dispersion among students by comparison-theory. The data rather support the prediction that dispersion of happiness tends to be lower among students, as implied in folklore-theory and livabilitytheory.

Scheme VI also shows that the size of the difference is rather variable across nations. In developed nations the differences are small to nonexistent; see e.g. Japan, the USA and Britain. Greater differences appear in developing nations such as South Africa, Brazil and Mexico. Dispersion of student-happiness is in fact unrelated to dispersion of generalpopulation-happiness: $r=-0.15$ (ns), respectively $r=+0.20$ (ns). These results are again in line with the predictions of folklore-theory and livability-theory, but contradict the prediction derived from comparison-theory.

As we have seen in Scheme IV, dispersion of happiness is less strongly related to social inequality in the student survey, than among the general population studies. The correlations are weak and statistically insignificant among students, though typically in the same direction. This result is in line with the prediction of livability-theory.

The results of all the tests are summarized in the Schemes I and II. The predictions that were confirmed are marked with ' + ', the predictions that were contradicted with ' - '. The predictions that were neither confirmed nor rejected by the data are marked with ' \pm '.

\section{DISCUSSION}

The crux of this way of theory testing is of course in the inference of predictions. As we have seen above, inference is not without problems. Predictions depend very much on additional assumptions; by introducing different assumptions, one can predict almost any outcome. Does this mean this way of theory testing is senseless, or even that these theories cannot be falsified at all?

All three theories considered here are characterized by one central premise. In most cases these premises alone allowed a prediction. Some predictions are based on additional assumptions indeed. These cases are marked with '*' in the Schemes I and II.

The few additional assumptions were not picked from the blue. One is central in the application of the theory to the problem this inquiry started with; the question whether a better society breeds happier people. This is the assumption that standards of comparison adjust to success. Some other additional assumptions are fairly evident, for instance the assumption of greater homogeneity among students. All the additional assumptions introduced here are falsifiable.

The predictions derived from comparison-theory are all defied by the data. Most predictions 
derived from folklore-theory are not supported either. On the other hand, the predictions of livability-theory fit the data very well. The latter theory is thus clearly the best one.

The poor performance of both comparison-theory and folklore theory is probably the result of a common theoretical flaw. Both these theories are one-sided cognitive theories of happiness that ignore the affective-motivational part of human nature. Happiness is seen as an accidental by-product of arbitrary mental constructs, rather than a functional biopsychological compass that draws on affective as well as cognitive cues. Elsewhere I have considered the defects of cognitive theory in more detail (Veenhoven 1991: 25-30). Here it suffices to note that livability-theory does not labor these shortcomings.

As noted in Paragraph 2, livability-theory of happiness came under question when surveys in western nations showed that happiness is hardly related to socio-economic variables such as income, class, sex and age. These findings where interpreted as showing that 'subjective' appraisals of life are largely independent of its 'objective' quality. The present study shows a sizable relationship at the nation-level. Does that mean that subjective- and objective QOL correspond only across nations, but not within nations?

A first thing to note in this context, is that there are clear subjective-objective relationships within nations. In poor and unequal nations we find typically strong correlations between happiness and income, and between happiness and class. In all nations we find correlations with occupation; managers and professionals being typically the happiest everywhere. In western nations there are also sizable relationships with marital status; the married being happier than the single. For a review see Veenhoven (1984).

A second point is that a low subjective-objective correlation in some condition does not falsify livability-theory. As livability-theory presumes that happiness results from needgratification it can explain such observations as satiation. Low correlations between happiness and income an affluent welfare states is then the result of diminished marginal utility of money.

\section{CONCLUSION}

The cross-national pattern of happiness cannot be predicted on the basis of comparisontheory, and neither by folklore-theory. Livability-theory predicts the observed pattern quite well. Apparently this common sense theory is the most realistic one. 


\section{REFERENCES}

Balatsky, G. and E. Diener (1993),

Subjective well-being among Russian students,

Social Indicators Research 28, pp. 225-244.

Brickman, P. and D. T. Campbell (1971),

Hedonic relativism and planning the good society.

in Appley (ed.), Adaptation Level Theory, a Symposion, Academic Press, London. UK, pp. $287-302$.

Easterlin, R. A. (1974),

Does economic growth improve the human lot? Some empirical evidence,

in P. A. Davis and W. R. Melvin (eds.), Nations and Households in Economic Growth, Stanford University Press, Paolo Alto, pp. 98-125.

Estes, R. J. (1984),

The Social Progress of Nations

Preager, New York.

IMF (1987),

Government Finance Statistics Yearbook, Vol XI

Intemational Monetary Fund, Washington.

Inglehart, R. (1990),

Culture Shift in Advanced Industrial Society

Princeton University Press, New York.

Kurian, G. T. (1979),

The Book of World Rankings

McMillan Reference Books, London.

Michalos, A. C. (1985),

Multiple Discrepancies Theory (MDT),

Social Indicators Research 16. pp. 347-413.

Michalos, A.C. (1991),

Global report on student well-being,

Vol I Life-satisfaction and Happiness, Springer Verlag, New York.

Ostroot, N. and W. W. Snyder (1985),

Measuring cultural bias in a cross-national study,

Social Indicators Research 17, pp. 234-251.

Summers, R. and A. Heston (1988),

A new set of international comparisons of real product and price level estimates for 130 countries, 1950-1985,

National income and Wealth 34. pp 1-25. 
Unger, H. E. (1971).

The feeling of happiness,

Psychology 7, pp. 27-33.

UN (1985).

National Account Statistics,

Compendium of Income Distribution Statistics, United Nations, Washington.

UNPD (1990).

World Development Report 1990

United Nations Development Programme, Washington.

Veenhoven, R. (1984),

Conditions of Happiness

Kluwer Academic, Dordrecht.

Veenhoven. R, (1991).

Is happiness relative?

Social Indicators Research 24, pp. 1-34.

Veenhoven. R. (1993A),

Happiness as an indicator in social policy evaluation: some objections considered,

in K. Mesman Schultz et al. (eds.), Between Sociology and Sociological Practice, Institute for Applied Social Sciences, Nijmegen pp. 195-206.

Veenhoven, R. (1993B),

Happiness in Nations, Subjective Appreciation of Life in 55 Nations 1946-1990, RISBO, Erasmus University Rotterdam.

Veenhoven, R. (1994),

Is happiness a trait?

Social Indicators Research, in press. 


\section{Scheme I}

\section{Predictions on Happiness in Nations Implied in Three Theories}

Predictions about level and dispersion Theories of happiness

of happiness in nations

$$
\text { Comparison-theory Folklore-theory Livability-theory }
$$

\section{Level of happiness in nations}

prevalent level

similarity across nations

correspondence with quality of life in nations

$\begin{array}{cccccc}\text { neutral } & - & & & & \\ \text { high } & - & \text { low* } & + & \text { low } & + \\ \text { low } & - & \text { low } & - & \text { high } & +\end{array}$

\section{Dispersion of happiness in nations}

prevalent dispersion

$\begin{array}{llllll}\text { small } & - & \text { small } & - & & \\ \text { high } & - & \text { high } & - & \text { low } & + \\ \text { low } & - & \text { low } & - & \text { high } & +\end{array}$

similarity across nations

correspondence with inequality in nations

$+=$ prediction confirmed $-=$ prediction disconfirmed $*=$ prediction based on additional assumption 


\section{Scheme II}

\section{Predictions about Differences in the Cross National Pattern of Happiness Implied in Three Theories}

Predictions about differences in happiness pattern between university-students and average citizens in nations
Theories of happiness

Comparison-Theory Folklore-theory Livability-Theory
Differences in level of happiness

prevalent level

similarity across nations

correspondence with quality of life in nations

\section{Differences in dispersion of happiness}

prevalent dispersion

similarity across nations

correspondence with inequality in lifechances in nations

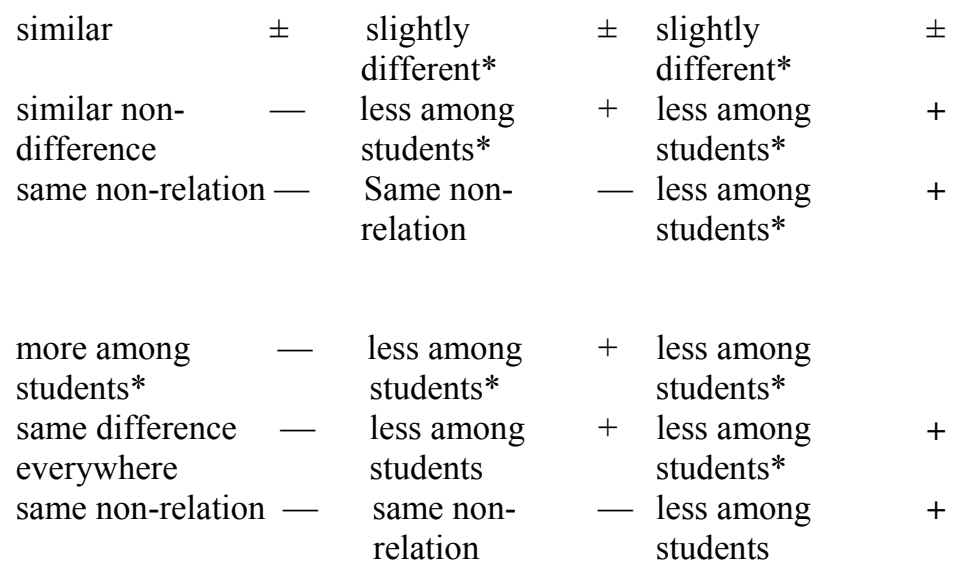

$+=$ prediction confirmed $-=$ prediction disconfirmed $\pm=$ results unclear $*=$ prediction based on additional assumption 


\section{Scheme III}

Average happiness and quality-of-life in nations in the 1980's

\begin{tabular}{|c|c|c|}
\hline \multirow[t]{3}{*}{ Quality-of-life } & \multicolumn{2}{|c|}{ Correlation with average happiness } \\
\hline & university students & general population \\
\hline & $\mathrm{N}$ & $\mathrm{N}$ \\
\hline
\end{tabular}

\begin{tabular}{|c|c|c|c|}
\hline $\begin{array}{l}\text { Material comfor } \\
\text { nutrition }\end{array}$ & +0.16 & 33 & +0.28 \\
\hline $\begin{array}{l}\text { real income per } \\
\text { capita }\end{array}$ & $+0.55 * *$ & 33 & $+0.69 * *$ \\
\hline $\begin{array}{l}\text { Social equality } \\
\text { social security }\end{array}$ & $+0.38 *$ & 32 & $+0.51 *$ \\
\hline gender equality & $+0.38 *$ & 34 & $+0.61 *$ \\
\hline Income equality & -0.08 & 24 & +0.22 \\
\hline
\end{tabular}

\section{Political freedom}

freedom of press $+0.63 * * \quad 33+0.54 * * \quad 28$

political democracy $+0.59 * * 24 \quad+0.58 * 24$

Access to knowledge

$\begin{array}{lllll}\text { education } & +0.50^{* *} & 34 & +0.69 * * & 28 \\ \text { media attendence } & +0.49 * & 30 & +0.55^{*} & 27\end{array}$

Explained variance $\left(\mathrm{R}^{2}\right) 0.57 \quad 20 \quad 0.78^{*} \quad 24$

Data: Happiness: Appendix A (happiness); QOL: Nutrition (caloric intake), UNDP (1990); Real income pc, Summers \& Heston (1988: 125): Social security expenditures \% gnp, reversed), IMF (1987); Gender equality (womens status, reversed), Estes (1984: 184-5); Income equality (Ginicoefficients, reversed) UN (1985); Freedom of press (1970's), Kurian (1979: 362); Political Democracy, Estes (1984, 175-187); Education, Estes (1984: 169, 183-4); Media attendance (1970's), Kurian (1979: $347-359)$.

$*=\mathrm{p}<0.05 \quad * *=\mathrm{p}<0.01$ 


\section{Scheme IV}

Dispersion of happiness and inequality in nations in the $1980 \mathrm{~s}$

Indicators of social inequality in nations Correlation with disperson (SD) of happiness in nations

\begin{tabular}{|c|c|c|c|c|}
\hline & \multicolumn{2}{|c|}{ university studens } & \multicolumn{2}{|c|}{ general population } \\
\hline & r & $\mathrm{N}$ & $\mathrm{r}$ & $\mathrm{N}$ \\
\hline $\begin{array}{l}\text { Income inequality } \\
\text { (Gini-coefficients) }\end{array}$ & -0.06 & 24 & $+0.64 * *$ & 25 \\
\hline $\begin{array}{l}\text { Social (in)security } \\
\text { (low expenditures) }\end{array}$ & +0.11 & 32 & $+0.41^{*}$ & 27 \\
\hline $\begin{array}{l}\text { Gender inequality } \\
\text { (large male-female differences) }\end{array}$ & +0.05 & 34 & +0.28 & 28 \\
\hline $\begin{array}{l}\text { Political inequality } \\
\text { (low democracy) }\end{array}$ & +0.09 & 24 & $+0.54 * *$ & 24 \\
\hline Variance explained $\left(\mathrm{R}^{2}\right)$ & 0.18 & 20 & $0.63 * *$ & 22 \\
\hline
\end{tabular}

Data; Happiness. Appendix B (happiness). Social Inequality:

Scheme IV.

$*=\mathrm{p}<0.05 \quad * *=\mathrm{p}<0.01$ 
Scheme V

Level of Happiness in Nations

Average scores on two items by students and general public in 23 nations in the 1980's

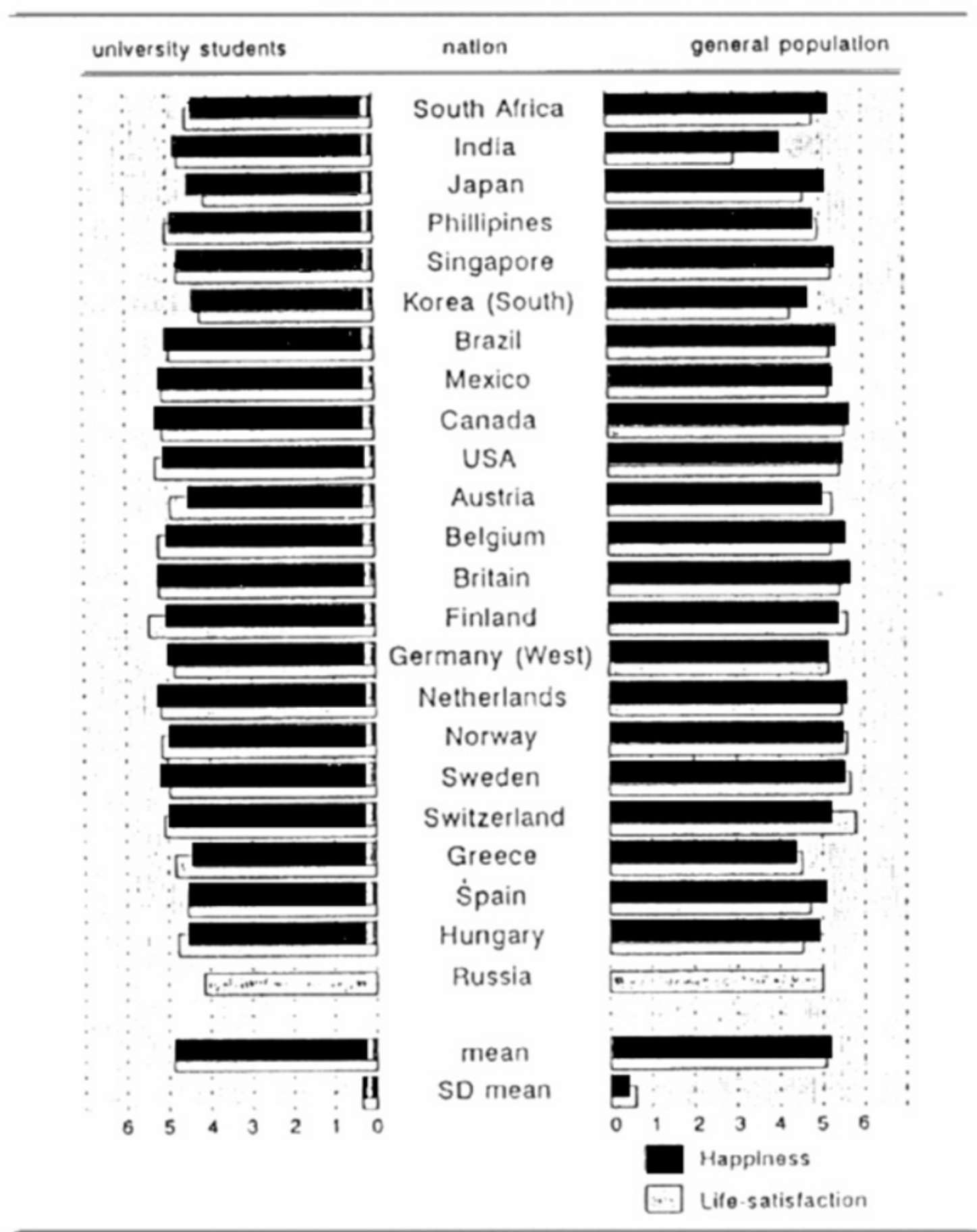

\section{Data: Appendix A}

Correspondence of average happiness in student data and general population data: Happiness, $r=+0.44(p<0.05)$, Life-satisfaction. $r=+0.56(p<0.01)$ 
Scheme VI

Dispersion of Happiness in Nations

Standard deviations on two items by students and general public

in 18 nations in the 1980's

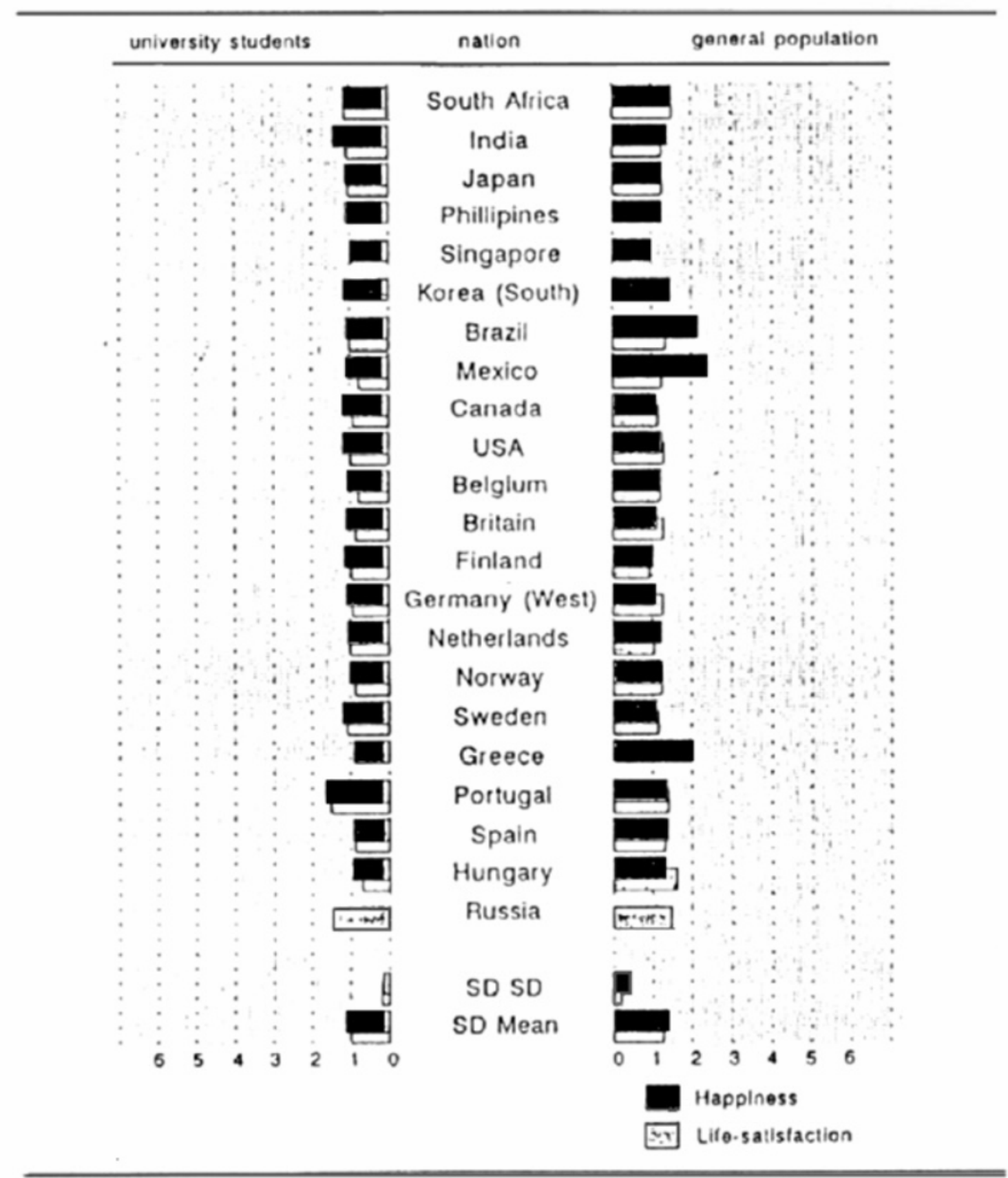

Data: Appendix B

Correspondence of dispersion of happiness in student data and general population data: Happiness, $r=+0.15$ (ns), Life-satisfaction, $r=+0.20$ (ns) 


\section{Appendix A}

\begin{tabular}{|c|c|c|c|c|c|}
\hline \multicolumn{6}{|c|}{$\begin{array}{c}\text { Level of Happiness in Nations in the 1980's } \\
\text { Average scores on scale } 1-7\end{array}$} \\
\hline \multirow[t]{2}{*}{ Nations by region } & & \multicolumn{2}{|c|}{ University students } & \multicolumn{2}{|c|}{ General population } \\
\hline & & happiness & lifesat & happiness & lifesat \\
\hline \multicolumn{6}{|l|}{ AFRICA } \\
\hline Cameroon & & 4.02 & 412 & & \\
\hline Kenya & & 4.46 & 4.46 & & \\
\hline South Africa & & 4.37 & 4.53 & 5.22 & 4.86 \\
\hline Tanzania & & 4.95 & 4.97 & & \\
\hline \multicolumn{6}{|l|}{ MIDDLE EAST } \\
\hline Egypt & & 4.44 & 4.55 & & \\
\hline Bahrain & & 4.68 & 4.96 & & \\
\hline Israel & $*$ & 4.58 & 4.98 & & \\
\hline Jordan & & 4.53 & 4.49 & & \\
\hline Turkey & & 4.61 & 4.21 & & \\
\hline \multicolumn{6}{|l|}{ FAR EAST } \\
\hline Bangaladesh & & 5.00 & 4.31 & & \\
\hline India & $*$ & 4.82 & 4.72 & 4.10 & 3.01 \\
\hline Japan & $*$ & 4.49 & 4.09 & 5.13 & 4.63 \\
\hline Philippines & $*$ & 4.90 & 5.02 & 485 & 4.96 \\
\hline Singapore & $*$ & 4.73 & 4.76 & 535 & 527 \\
\hline South Korea & & 4.38 & 4.21 & 4.73 & 4.30 \\
\hline Taiwan & & 4.56 & 4.35 & & \\
\hline Thailand & & 4.07 & 4.61 & & \\
\hline \multicolumn{6}{|l|}{ LATIN AMERICA } \\
\hline Brazil & $*$ & 5.04 & 4.94 & 5.36 & 5.22 \\
\hline Chile & & 4.70 & 4.97 & & \\
\hline Colombia & & 5.02 & 5.12 & & \\
\hline Mexico & $*$ & 5.17 & 5.12 & & \\
\hline Puerto Rico & & 5.02 & 5.18 & 5.26 & 5.18 \\
\hline \multicolumn{6}{|l|}{ ANGLO AMERICA } \\
\hline Canada & $*$ & 5.27 & 5.11 & 5.67 & 5.56 \\
\hline USA & $*$ & 5.06 & 5.28 & 5.51 & 5.45 \\
\hline \multicolumn{6}{|l|}{ AUSTRALIA } \\
\hline Australia & $*$ & & & 5.67 & 5.60 \\
\hline New Zealand & & 5.07 & 5.06 & & \\
\hline \multicolumn{6}{|l|}{$\begin{array}{l}\text { NORTH WESTERN } \\
\text { EUROPE }\end{array}$} \\
\hline Austria & $*$ & 4.48 & 4.92 & 5.01 & 5.26 \\
\hline Belgium & $*$ & 4.99 & 5.21 & 5.56 & 5.24 \\
\hline Britain & $*$ & 5.22 & 5.20 & 5.68 & 5.45 \\
\hline Denmark & $*$ & & & 5.63 & 5.75 \\
\hline Finland & $*$ & 5.01 & 5.44 & 5.37 & 5.61 \\
\hline Germay (West) & $*$ & 4.97 & 4.84 & 5.14 & 5.16 \\
\hline Ireland & & & & 5.72 & 5.55 \\
\hline Iceland & & & & 5.76 & 5.73 \\
\hline
\end{tabular}




\section{Appendix A continued}

\begin{tabular}{|c|c|c|c|c|c|}
\hline Netherlands & $*$ & 5.23 & 5.17 & 5.60 & 5.48 \\
\hline North Ireland & & & & 5.67 & 5.45 \\
\hline Norway & $*$ & 4.96 & 5.14 & 5.49 & 5.60 \\
\hline Luxembourgh & & & & 5.27 & 5.56 \\
\hline Sweden & $*$ & 5.19 & 4.96 & 5.54 & 5.68 \\
\hline Switzerland & $*$ & 4.95 & 5.08 & 5.21 & 5.80 \\
\hline \multicolumn{6}{|c|}{ SOUTHERN EUROPE } \\
\hline Croatia & & 4.54 & 4.70 & & \\
\hline France & $*$ & & & 5.36 & 4.77 \\
\hline Greece & & 4.42 & 4.83 & 4.37 & 4.54 \\
\hline Italy & $*$ & & & 4.89 & 4.74 \\
\hline Portugal & $*$ & 2.25 & 3.26 & 4.72 & 5.04 \\
\hline Spain & $*$ & 4.52 & 4.55 & 5.10 & 4.73 \\
\hline \multicolumn{6}{|c|}{ EASTERN EUROPE } \\
\hline Hungary & $*$ & 4.52 & 4.77 & 4.93 & 4.55 \\
\hline Russia & & & 4.15 & 4.60 & 5.01 \\
\hline Mean average & & 4.63 & 4.81 & 5.23 & 5.15 \\
\hline
\end{tabular}

Data: University students: Michalos 1991:83, General population: Veenhoven 1993

(happiness table 1.1.1 b, completed with data from tables 1.1.1 a and 1.1.1 c. Lifesatisfaction: tables 1.2.2 a $+\mathrm{b}$ completed with data front tables 1.2.1 $\mathrm{a}+\mathrm{h}+\mathrm{c}$ ). $0-10$ expert scores transformed linearly to $1-7$ )

Russia: Student data concern former USSR (Balatsky and Diener 1993)

General population data concern White Russia only

Portugal: Student data dubious; not included in analysis.

* Nations on which full QOL data are available. Used in analysis in Scheme III. 


\section{Appendix B}

\begin{tabular}{|c|c|c|c|c|c|}
\hline \multicolumn{6}{|c|}{$\begin{array}{c}\text { Level of Happiness in Nations in the 1980's } \\
\text { Average scores on scale } 1-7\end{array}$} \\
\hline \multirow[t]{2}{*}{ Nations by region } & & \multicolumn{2}{|c|}{ University students } & \multicolumn{2}{|c|}{ General population } \\
\hline & & happiness & lifesat & happiness & lifesat \\
\hline \multicolumn{6}{|l|}{ AFRICA } \\
\hline Cameroon & & 0.96 & 0.90 & & \\
\hline Kenya & & 1.11 & 1.01 & & \\
\hline South Africa & & 1.17 & 1.14 & 1.52 & 1.52 \\
\hline Tanzania & & 1.22 & 1.23 & & \\
\hline \multicolumn{6}{|l|}{ MIDDLE EAST } \\
\hline Egypt & & 1.12 & 1.06 & & \\
\hline Bahrain & & 1.30 & 1.22 & & \\
\hline Israel & $*$ & 1.39 & 0.85 & & \\
\hline Jordan & & 1.19 & 1.12 & & \\
\hline Turkey & & 1.09 & 1.02 & & \\
\hline \multicolumn{6}{|l|}{ FAR EAST } \\
\hline Bangaladesh & & 1.16 & 1.10 & & \\
\hline India & $*$ & 1.43 & 1.09 & 1.41 & 1.25 \\
\hline Japan & $*$ & 1.11 & 1.05 & 1.26 & 1.26 \\
\hline Philippines & $*$ & 1.11 & 0.92 & 1.25 & \\
\hline Singapore & $*$ & 1.00 & 0.95 & 0.99 & \\
\hline South Korea & & 1.17 & 1.50 & 1.47 & \\
\hline Taiwan & & 1.21 & 0.94 & & \\
\hline Thailand & & 1.05 & 0.94 & & \\
\hline \multicolumn{6}{|l|}{ LATIN AMERICA } \\
\hline Brazil & $*$ & 1.11 & 1.02 & 2.16 & 1.36 \\
\hline Chile & & 1.03 & 0.84 & & \\
\hline Colombia & & 1.10 & 0.86 & & \\
\hline Mexico & $*$ & 1.09 & 0.79 & 2.40 & 1.25 \\
\hline Puerto Rico & & 1.17 & 0.94 & & \\
\hline \multicolumn{6}{|c|}{ ANGLO AMERICA } \\
\hline Canada & $*$ & 1.20 & 0.94 & 1.08 & 1.15 \\
\hline USA & $*$ & 1.20 & 1.00 & 1.24 & 1.31 \\
\hline \multicolumn{6}{|l|}{ AUSTRALIA } \\
\hline Australia & $*$ & & & 1.08 & 0.88 \\
\hline New Zealand & & 1.22 & 0.96 & & \\
\hline \multicolumn{6}{|c|}{$\begin{array}{l}\text { NORTH WESTERN } \\
\text { EUROPE }\end{array}$} \\
\hline Austria & $*$ & 1.22 & 1.01 & 1.26 & \\
\hline Belgium & $*$ & 1.07 & 0.80 & 1.22 & 1.21 \\
\hline Britain & $*$ & 1.11 & 0.87 & 1.12 & 1.28 \\
\hline Denmark & $*$ & & & 1.06 & 1.24 \\
\hline Finland & $*$ & 1.16 & 0.99 & 1.02 & 0.94 \\
\hline Germay (West) & $*$ & 1.11 & 0.96 & 1.06 & 1.27 \\
\hline Ireland & & & & 1.20 & 1.26 \\
\hline Iceland & & & & 1.10 & 1.08 \\
\hline Netherlands & $*$ & 1.08 & 1.02 & 1.22 & 1.05 \\
\hline North Ireland & & & & 1.24 & 1.28 \\
\hline Norway & $*$ & 1.02 & 0.89 & 1.20 & 1.24 \\
\hline Luxembourgh & & & & 1.71 & \\
\hline Sweden & $*$ & 1.21 & 1.09 & 1.10 & 1.16 \\
\hline Switzerland & $*$ & 1.06 & 0.83 & 1.71 & \\
\hline
\end{tabular}




\section{Appendix B continued}

\begin{tabular}{|c|c|c|c|c|c|}
\hline \multicolumn{6}{|c|}{ SOUTHERN EUROPE } \\
\hline Croatia & & 1.22 & 1.14 & & \\
\hline France & $*$ & & & 1.20 & 1.41 \\
\hline Greece & & 0.92 & 0.90 & 2.01 & \\
\hline Italy & $*$ & & & 1.32 & 1.68 \\
\hline Portugal & & 1.66 & 1.52 & 1.36 & 1.41 \\
\hline Spain & $*$ & 0.94 & 0.89 & 1.38 & 1.33 \\
\hline \multicolumn{6}{|c|}{ EASTERN EUROPE } \\
\hline Hungary & $*$ & 0.96 & 0,72 & 1.32 & 1.61 \\
\hline Russia & & & 1.48 & 1.46 & 1.47 \\
\hline Mean SD & & 1.07 & 0.99 & 1.30 & 1.28 \\
\hline
\end{tabular}

Data:University students; Michalos 1991:83, General population: Veenhoven 1993.

- Happiness: table 1.1.1 b, completed with data from tables $1.1 .1 \mathrm{a}$ and $1.1 .1 \mathrm{c}$.

SD on scale $1-4$ (resp $1-3$ arid $1-5$ ) transformed linearly to $1-7$ )

- Life satisfaction: tables $1.2 .2 \mathrm{a}+\mathrm{b}$ : SD on scale $0-10$ or $1-10$ transformed linearly to $1-7$ expert scores transformed linearly to $1-7$.

Russia: Student data concem former USSR (Balatsky and Diener 1993).

- General population data concern White Russia only

Portugal: Student data dubious; not included in analysis.

* Nations on which full QOL data are available. Used in analysis in Scheme IV. 